\title{
Correction to: Heart rate variability, the dynamic nature of the retinal microvasculature and cardiac stress: providing insight into the brain-retina-heart link: the SABPA study
}

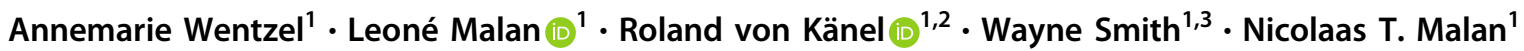

Published online: 4 September 2019

(c) The Author(s), under exclusive licence to The Royal College of Ophthalmologists 2019

\section{Correction to: Eye}

https://doi.org/10.1038/s41433-019-0515-y

Since the online publication of this article, the authors have noticed that the conclusion to the abstract contains an error. The correct abstract conclusion should read as follows:

FLIP elicited increased SNS activity and modulation in this bi-ethnic cohort. In Africans, decreased HRV during
FLIP accompanied arteriolar and venular dilatory responses and elevated systemic levels of cTnT, implying that the SNS exerted a significant effect on the smooth muscle tone of the retinal vasculature. Disrupted retinal autoregulation may imply general autonomic nervous system dysfunction; exemplifying central control by the brain on all systemic regulatory functions, across different vascular beds.

The authors apologise for any inconvenience caused.

Leoné Malan

Leone.Malan@nwu.ac.za

1 Hypertension in Africa Research Team (HART), North-West

University, Potchefstroom, South Africa

2 Department of Consultation-Liaison Psychiatry and Psychosomatic Medicine, University Hospital Zurich, Zurich, Switzerland

3 South African Medical Research Council, Unit for Hypertension and Cardiovascular Disease, North-West University,

Potchefstroom, South Africa 